\title{
Short-Term Effects of a Resistance Training Program Using Elastic Tubing in Patients with Heart Disease
}

João Pedro Lucas Neves Silva, ${ }^{\circledR}$ Tamara lasmin de Sá Ferreira, ${ }^{\circledR}$ Gabriela Côrtes Cavalleri, ${ }^{\circledR}{ }^{\circledR}$ Mayara Moura Alves da Cruz, ${ }^{10}$ Bianca Pinhal Galindo, ${ }^{2}$ Natália Turri da Silva, ${ }^{3}$ Bruna Spolador de Alencar Silva, ${ }^{\circledR}$ Marceli Rocha Leite, ${ }^{1 \oplus}$ Ana Paula Coelho Figueira Freire, ${ }^{2}$ Ercy Mara Cipulo Ramos, ${ }^{\circledR}$ Luiz Carlos Marques Vanderlei, ${ }^{\circledR}$ Francis Lopes Pacagnelli ${ }^{2}{ }^{\circledR}$

Universidade do Estado de São Paulo (UNESP), ${ }^{1}$ São Paulo, SP - Brazil.

Universidade do Oeste Paulista (UNOESTE), ${ }^{2}$ Presidente Prudente, SP-Brazil.

Universidade de Brasília, Brasília, DF - Brazil.

\section{Abstract}

Background: Resistance training is effective in cardiac rehabilitation; however, it is conventionally performed using free weights or machines, which can pose logistic challenges to patients with restricted mobility. For its ease of access and cost-effectiveness, elastic tubing is a particularly appealing alternative, but it remains underutilized for this purpose.

Objective: To evaluate muscle strength, functional capacity, aerobic capacity, and quality of life in patients with heart disease in phase II of cardiovascular rehabilitation after a resistance training intervention based solely on elastic tubing.

Methods: Thirteen patients with heart disease (age $63.33 \pm 10.80$ years) trained with elastic tubing twice weekly for 6 weeks, with progressive load increase every 15 days. The following muscle groups were evaluated and trained: shoulder abductors and flexors, elbow flexors, and knee flexors and extensors. Muscle strength was evaluated using a dynamometer; functional capacity, with a 6-minute walk test and cardiopulmonary exercise test; and quality of life, using the SF-36 questionnaire. Data normality was assessed using the Shapiro-Wilk test. The paired Student's t-test was used for comparisons before and after training, at a significance level of $<5 \%$.

Results: There were significant differences in muscle strength (except for elbow flexion) and functional capacity (485.5 \pm 123.3 vs $578.7 \pm 110.5 ; p=0.0399$ ) after the intervention. No statistical differences were found in cardiorespiratory fitness or quality of life.

Conclusions: Short-term resistance training with elastic tubing improved peripheral muscle strength and functional capacity in patients with heart disease, and should be encouraged for this population. (Int J Cardiovasc Sci. 2021; 34(2):149-156)

Keywords: Resistance Training; Exercise; Cardiovascular Diseases; Muscle Strength Dynamometer; Functional Residual Capacity; Cardiac Rehabilitation; Elastic Tubing.

\section{Introduction}

Approximately 7.3 million deaths per year occur worldwide as a result of cardiovascular disease (CVD), a number that is expected to exceed 23.6 million by 2030. ${ }^{1}$ In Brazil, 300,000 people die each year from CVD, including stroke, heart failure, and heart attack or sudden cardiac death, representing 820 deaths per day, 30 deaths per hour, or one death every 2 minutes.,3

Heart diseases result in physical deconditioning and skeletal muscle changes, such as atrophy and decreased strength, that interfere with functional capacity and 
quality of life., ${ }^{4,5}$ Therefore, it is of paramount importance that individuals with heart disease be included in a cardiac rehabilitation program (CRP). ${ }^{5}$

A CRP consists of four phases, with the first beginning in hospital, and is an ongoing process which aims to maintain well-being and facilitate resumption of social and professional activities. ${ }^{6,7}$ Phase 2 of cardiac rehabilitation begins after hospital discharge or a few days after a cardiovascular event. At this stage, resistance training can be initiated to increase fatigue resistance and muscle strength, with a view to return to work or recreational activities.

In clinical practice, resistance training to improve muscle strength is conventionally performed by means of free weights or weight machines, however, these require extensive physical space and are usually located at outside facilities, such as gyms and rehabilitation centers, which poses an additional challenge to patients with restricted mobility. ${ }^{4}$ On the other hand, elastic tubes are practical, low-cost, portable, safe devices which can be used for resistance training, with the added advantage of increasing tension linearly from the start of the contraction until the end of the movement, which tends to be less damaging to the joints compared to freeweight or weight machine training. ${ }^{10,11}$

Training with elastic tubing has been associated with increased muscle strength in older adults with heart and lung diseases, ${ }^{4,12}$ and has been shown to promote cardiorespiratory improvement in patients with chronic obstructive pulmonary disease. ${ }^{13}$ However, whether this exercise modality can have a positive impact on cardiorespiratory capacity and quality of life in patients with heart disease remains unclear. ${ }^{12}$ We hypothesized that this alternative method of strength training would yield short-term gains in muscle strength, functional ability, aerobic capacity, and quality of life of these patients.

Thus, the objective of this study was to analyze the effects of a strength training program based on elastic tubing on functional capacity, maximum aerobic capacity, muscle strength, and quality of life in individuals with CVD in phase 2 of cardiovascular rehabilitation.

Methods

\section{Case Series}

This was a clinical trial conducted at a school of Physical Therapy. Male and female patients aged 45-79 years, with known heart disease (coronary artery disease, status post myocardial revascularization procedures, or acute myocardial infarction) who had been in phase 2 of cardiac rehabilitation for at least 3 months were recruited.

For participation, patients were required to be hemodynamically stable (mean arterial pressure 90-120 $\mathrm{mmHg}$ and no arrhythmia of any type), with no changes in medication for a minimum of 30 days. All patients were instructed not to perform resistance training with weights, dumbbells, or any device other than the elastic tubing. Patients with neurological, musculoskeletal, or articular disorders (such as rheumatic disorders), unstable heart disease as detected by electrocardiography, or lung diseases which might prevent them from completing the training protocol were excluded.

To avoid outcome bias, assessments and training sessions were conducted by different therapists. The following variables were evaluated: muscle strength, functional capacity, maximum aerobic capacity, and quality of life before and after 6 weeks of resistance training with elastic tubing.

All participants were given information regarding study procedures and provided written informed consent before enrollment. The study protocol was approved by the institutional Research Ethics Committee (CAAE: 44443915.5.0000.5515) and was registered on the ClinicalTrials.gov platform under identifier code NCT03580538.

\section{Evaluation of Muscle Strength}

Muscle strength was evaluated at the start of the intervention protocol and after 6 weeks, using a digital force gauge (Force Gauge ${ }^{\circledR}$, model FG-100kg, United States), and all results were expressed in newtons (N). The evaluation was performed using a steel cord coated with rigid plastic attached to a dynamometer, with one end attached to a fixed bar and the other end to the distal portion of the dominant limb of the patient. ${ }^{14}$

The following muscle groups were evaluated: shoulder abductors and flexors, elbow flexors, and knee extensors and flexors. The measurement was carried out in triplicate for each proposed movement, and the highest value of the three repetitions was recorded. During evaluation, the patient was required to maintain the maximum isometric contraction achieved for a period of 6 seconds, after which time they were allowed to relax their muscles for 1 minute before a new measurement was performed. Strength of the upper limbs was 
measured with the patient in the standing position, and that of the lower limbs, with the patient sitting in the same chair used for resistance training. ${ }^{14}$

\section{Evaluation of Functional Capacity}

Functional capacity was evaluated by the 6-minute walk test (6MWT), conducted as per American Thoracic Society recommendations. ${ }^{15}$ The test was performed in a gymnasium and was repeated twice, with an interval of 30 minutes between attempts. The highest value was taken into account for analysis. During the test, standardized verbal performance incentives were given every minute. Blood pressure, pulse oximetry, subjective perception of exertion, and heart and respiratory rate were measured throughout.

\section{Evaluation of Maximal Aerobic Capacity}

Cardiopulmonary exercise testing (CPET) was performed by a cardiologist. All tests were performed on a treadmill (Inbrasport ATL 2000). The modified Bruce protocol ${ }^{16}$ was used, and the test performed until voluntary exhaustion. No patient exhibited any electrocardiographic alterations that might prevent completion of the test.

Cardiovascular parameters were monitored continuously: heart rate (Polar S810i, Finland), arterial oxygen saturation (Mindray PM 50, Brazil), and subjective perception of exertion (modified Borg scale). ${ }^{17}$ The ventilatory variables were obtained through a VO2000 gas analyzer (Medical Graphics, USA), calibrated before each test according to the manufacturer's instructions. The average air flow, obtained every 10 seconds (Aerograph $®$, Michigan, USA), was used for all tests.

Peak oxygen consumption $\left(\mathrm{VO}_{2 \text { peak }}\right)$ was calculates as the highest average maximum oxygen consumption $\left(\mathrm{VO}_{2}\right)$ of the final 30 seconds of evaluation, when at least two of the following criteria were met: 1) heart rate $>90 \%$ of maximum predicted for the age of each patient $(220$ - age); 2) respiratory quotient $(\mathrm{RQ})>1.10$; 3) variation in $\mathrm{VO}_{2}$ between the final two stages of evaluation less than $2.1 \mathrm{ml} \cdot \mathrm{kg}^{-1} \cdot \mathrm{min}^{-1}$. The velocity corresponding to $\mathrm{VO}_{2 \text { peak }}\left(\mathrm{vVO}_{2 \text { peak }}\right)$ was recorded as the maximum intensity achieved during the test. If the patient reached exhaustion before the completion of the stage, the $\mathrm{vVO}_{2 \text { peak }}$ was adjusted according to the equation proposed by Kuipers et al., ${ }^{18}$

\section{Evaluation of Quality of Life}

Quality of life was assessed with the SF-36 generic questionnaire, validated for use in Brazil. The SF-36 consists of eight dimensions, scored from 0 to 100. For each dimension, values greater than 50 denote perception of good quality of life. ${ }^{19}$

\section{Cardiovascular Parameters}

Before and after each training session, the following cardiovascular parameters were recorded: systolic blood pressure (SBP), diastolic blood pressure (DBP), and heart rate (HR).

Blood pressure was measured in the dominant arm, with the participant in the seated position, using an aneroid sphygmomanometer (Premium $\AA$, China) and stethoscope ( $3 \mathrm{M}^{\mathrm{TM}}$ Littmann ${ }^{\circledR}$, USA), following the latest Brazilian hypertension guidelines. ${ }^{20} \mathrm{HR}$ was monitored using a Polar@ monitor (Polar, USA).

\section{Resistance Training}

Training sessions (duration $60 \mathrm{~min}$ each) took place in a climate-controlled environment, twice weekly over six weeks, for a total of 12 sessions. For safety, training heart rate reserve was calculated individually by the Karvonen formula. ${ }^{21,22}$ During training, patients could not exceed this heart rate. The Borg scale was also used to control exercise intensity. ${ }^{23}$

Each training session was preceded by measurement of heart rate and blood pressure, followed by stretching of the upper and lower limbs. During the training period, patients performed only resistance training, not aerobic training. The resistance exercises were performed using Lemgruber@ brand latex tubing (references: 200, 201, 202, 203, and 204), as well as an appropriate chair (see below).

Each reference number denotes different internal and external diameters, with higher numbers indicating higher resistance when the tube is taut; i.e., lower reference numbers imply a lower load for the patient, while higher references imply a higher load. The resistance is increased when more elastic tubing is added in subsequent sessions.

The muscle groups selected for training, on the basis of a previous study, were the shoulder abductors and flexors, elbow flexors, and knee extensors and flexors. The length of elastic tubing was determined according to the individual distance from the upper or lower limb to the hook on the chair. The upper-limb and lower- 
limb movements were performed with the patient in the sitting position, with the exception of knee flexion, in which the patient stood in front of the chair used in the dynamometer test. As the elastic tubes should be fixed during training, chairs were constructed especially for this purpose; each chair was $72 \mathrm{~cm}$ high and $52 \mathrm{~cm}$ wide, and had points of attachment for the elastic tubes depending on the muscle group being exercised. For this purpose, one end of the elastic tube is fixed to the segment of the body that performs the arc of movement and the other is attached to the chair support. All movements were performed alternately and bilaterally throughout the permitted range of motion of each joint, with a 2-minute interval between series. ${ }^{13}$

The training protocol and increase in resistance (table 1), given according to the reference number of the elastic tubing, was evaluated individually based on the Borg rating of perceived exertion (13, slightly tiring). ${ }^{22}$

\section{Statistical Analysis}

Sample size calculation was based on the peripheral muscle strength of knee extension in a previous study by Ramos et al., ${ }^{14}$ with a standard deviation of 36.62, difference to be detected of 45.1 , significance level of $5 \%$, and test power of $80 \%$. The sample size was calculated as 10 subjects per group.

The Shapiro-Wilk test was used to verify the assumption of normality. As all data were considered normal, Student's $t$-test for paired data was used for comparisons before and after resistance training, with results expressed as mean \pm standard deviation. A significance level of $<0.05$ was established. All analyses were carried out in GraphPad Prism ${ }^{\circledR}$.

\section{Results}

Due to the difficulty of adherence and the musculoskeletal limitations of some patients presented, only 13 subjects with heart disease were evaluated. One patient left the study due to hospitalization. Maximum aerobic capacity data (Table 3) is only available for eight patients, due to failure to capture data during one CPET at the final time point of evaluation. Data on functional capacity were excluded from one patient who experienced ankle pain.

The sample consisted of 12 patients with heart disease, 8 men and 4 women, with a mean age of $63.33 \pm 10.80$ years. The mean weight, height, and BMI were $77.35 \pm 13.95 \mathrm{~kg}, 1.60 \pm 0.07 \mathrm{~m}$, and $29.92 \pm 4.28 \mathrm{~kg} /$ $\mathrm{m}^{2}$ respectively. The most commonly used drugs were antihypertensives $(n=6)$, antiplatelet agents $(n=6)$, and antilipemics $(n=8)$. The most prevalent comorbidities were hypertension, diabetes, coronary heart disease, and a history of myocardial revascularization .

Figure 1 shows the differences in peripheral muscular strength between the baseline and week 6 time points. Significant differences were found after resistance training with elastic tubing. In the lower limbs, significant increases in strength were observed during both knee extension and flexion, while in the upper limbs, a significant difference was noted only in shoulder abduction.

Significant differences in 6MWT were also found between the baseline and 6-week time points. These analyses are described in Table 2.

Table 3 shows the maximum aerobic capacity values of the evaluated patients. Analysis of $\mathrm{VO}_{2 \text { peak }}$ (in $\mathrm{mL} \cdot \mathrm{kg} / \mathrm{min}$ and $\mathrm{L}$ ) and $\mathrm{vVO}_{2 \text { peak }}$ did not demonstrate a significant increase after 6 weeks of training with elastic tubing.

Table 1 - Incremental change in resistance over the course of the training protocol.

\begin{tabular}{|c|c|c|c|}
\hline \multirow{2}{*}{ Time points } & \multicolumn{3}{|c|}{ Variables } \\
\hline & Repetitions & Reference UL & Reference LL \\
\hline 1st and 2nd week & $2 \times 15$ & 200 & 201 \\
\hline 3nd and 4th week & $2 \times 12$ & $200+201$ to $201+203$ & $201+202$ to $202+204$ \\
\hline 5th and 6th week & $3 \times 10$ & $200+202$ to $203+204$ & $\begin{array}{c}201+202+203+204 \\
\text { to all references }\end{array}$ \\
\hline
\end{tabular}




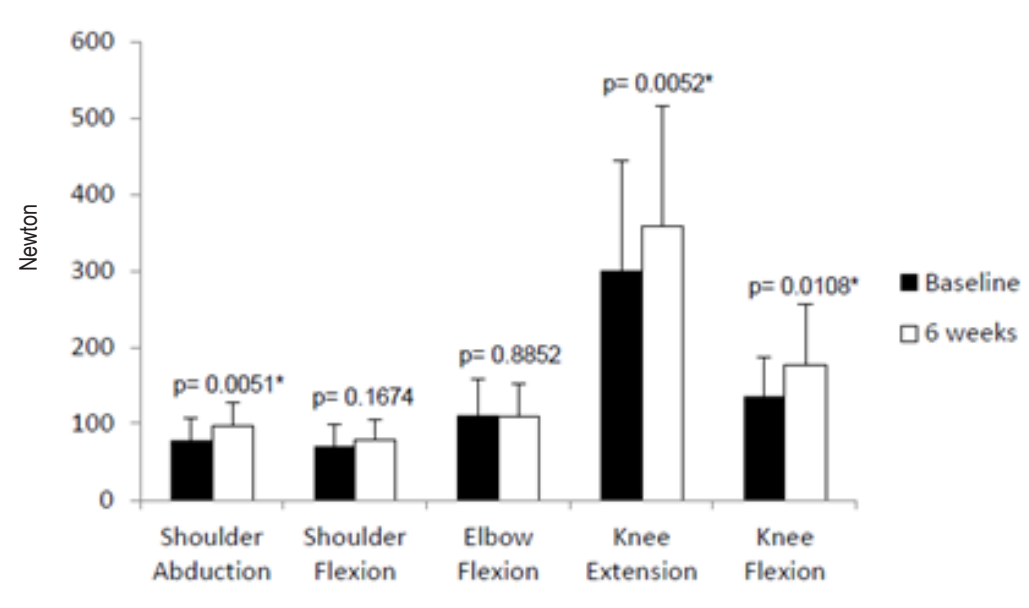

Figure 1 - Peripheral muscle strength presented as mean and standard deviation of the evaluated patients before and after 6 weeks of resistance training with elastic tubing. *Significant difference between the baseline moment and after 6 weeks of training. ShapiroWilk test followed by Student $t$ test.

Table 2 - Functional capacity through the 6-minute walk test of the evaluated patients.

\begin{tabular}{lcc}
\hline \multirow{2}{*}{ Variables } & \multicolumn{2}{c}{ Moments (n=11) } \\
\cline { 2 - 3 } & Baseline & p weeks \\
\hline Distance traveled (m) & $485.50 \pm 123.30$ & $578.70 \pm 110.50$ \\
\hline Distance Predicted (m) & $531.10 \pm 126.30$ & $531.10 \pm 126.30$ \\
\hline$M-$ meters. The results are presented as mean \pm standard deviation. Shapiro-Wilk test followed by Student t test. \\
\hline
\end{tabular}

The distribution of SF-36 quality of life scores is shown in Table 4. There was no significant difference in any of the eight dimensions of the questionnaire after 6 weeks of training with elastic tubing as compared to baseline.

\section{Discussion}

The present study showed that 6 weeks of resistance training with elastic tubing had a positive influence on muscle strength of the lower limbs and shoulder abductors, as well as on functional capacity, in patients with heart disease. However, there was no significant difference in maximum aerobic capacity or quality of life.

A gain in muscle strength is an expected result of resistance training, ${ }^{24}$ and is of great clinical relevance, as it is related to improved ability to perform activities of daily living and work in patients with heart disease. Moreover, in the specific population of patients with CVD, improved muscle strength is associated with less overload on the cardiovascular system.${ }^{14}$ One previous study of resistance training with elastic tubing demonstrated this effect of increasing muscle strength and increasing functional capacity in patients with heart disease. ${ }^{4}$ The authors compared the use of a resistance training program based on elastic tubing and conventional weight training. The intensity of resistance with elastic tubing was varied by changing the elastic tube length in relation to the body segment of the patient under evaluation, which differs from the present study, where resistance was increased by using different diameters of elastic tubing; the studies also followed different training schedules. ${ }^{25}$ 
Table 3 - Aerobic power of the evaluated patients.

\begin{tabular}{|c|c|c|c|}
\hline \multirow{2}{*}{ Variables } & \multicolumn{2}{|c|}{ Moments $(n=8)$} & \multirow[t]{2}{*}{ p-value } \\
\hline & Baseline & 6 weeks & \\
\hline $\mathrm{VO}_{2 \text { peak }}(\mathrm{mL} \cdot \mathrm{kg} / \mathrm{min})$ & $12.19 \pm 2.92$ & $14.70 \pm 5.12$ & 0.1200 \\
\hline $\mathrm{VO}_{2 \text { peak }}(\mathrm{L})$ & $0.94 \pm 0.32$ & $1.17 \pm 0.59$ & 0.1186 \\
\hline $\mathrm{vVO}_{2 \text { peak }}(\mathrm{km} / \mathrm{h})$ & $3.12 \pm 0.58$ & $3.54 \pm 0.66$ & 0.0533 \\
\hline
\end{tabular}

Table 4 - Quality of life of the evaluated patients as assessed by SF-36 domains.

\begin{tabular}{|c|c|c|c|}
\hline \multirow{2}{*}{ Dimensions } & \multicolumn{2}{|c|}{ Moments (n=10) } & \multirow{2}{*}{ p-value } \\
\hline & Baseline & 6 weeks & \\
\hline Functional capacity & $76.00 \pm 14.10$ & $74.50 \pm 4.80$ & 0.3898 \\
\hline Physical functioning & $41.67 \pm 50.00$ & $52.50 \pm 47.8$ & 1.0000 \\
\hline Pain & $63.33 \pm 22.88$ & $56.60 \pm 22.69$ & 0.4407 \\
\hline General health & $58.28 \pm 22.57$ & $56.80 \pm 13.31$ & 0.9702 \\
\hline Vitality & $63.89 \pm 14.31$ & $59.50 \pm 19.5$ & 0.7759 \\
\hline Role functioning, social & $78.33 \pm 25.59$ & $88.00 \pm 17.35$ & 0.2228 \\
\hline Role functioning, emotional & $55.51 \pm 47.16$ & $46.20 \pm 45.00$ & 0.6250 \\
\hline Mental health & $72.89 \pm 13.38$ & $68.70 \pm 20.18$ & 0.9174 \\
\hline
\end{tabular}

In relation to the initial resistance of the elastic tubing, Turban et al., ${ }^{4}$ assessed the one-repetition maximum (1RM), unlike in our study, where increasing resistance was measured according to perceived exertion on the Borg scale. Turban et al., ${ }^{4}$ reported improvement in functional capacity and increased muscle strength and noted that this training modality may be a tool with which to begin cardiac rehabilitation, not excluding the use of conventional equipment, but as an adjunct.

Ramos et al., ${ }^{14}$ compared resistance training performed with elastic tubing versus training carried out with a conventional weight machine in patients with COPD.
The elastic resistance protocol used individualized repetitions according to the fatigue resistance test; series started with two repetitions and progressed to seven at the end of the intervention, while the conventional training protocol consisted of three sets of 10 repetitions. Both training programs provided benefits in muscle strength and quality of life, however, greater gains in functional capacity were found in the elastic tubing group. ${ }^{14}$

Some important differences in protocol between the aforementioned study of COPD patients and the present study must be mentioned; for example, those authors used 8 weeks of training, while the present 
study used 6 weeks. Another difference concerns the way in which resistance and the number of repetitions were increased. In the present study, a gradual increase in resistance and series and a decrease in number of repetitions were implemented, so that the training was designed to increase muscular strength and not just fatigue resistance, as in the study of COPD patients. ${ }^{14}$

Regarding functional capacity, our participants exhibited a significant improvement after 6 weeks of resistance training. A recent meta-analysis reported similar behavior: conventional resistance with weight machines was associated with a significant gain in functional capacity as soon as 4 weeks after the start of training. ${ }^{26}$ Ramos et al., ${ }^{14}$ observed greater effects on functional capacity in training with elastic tubing when compared to conventional training, which demonstrates an advantage of this resource.

This gain in functional capacity observed in these studies may be explained by an increase in type I muscle fiber area and in oxidative enzyme activity in the skeletal musculature, both of which are known to be promoted by resistance training. ${ }^{27}$

The aerobic capacity of these patients did not demonstrate any significant change. This finding may be related to the lack of aerobic activity during the study training protocol. It is known that both the practice of aerobic exercise in isolation and aerobic exercises combined with resistance training promote improvement in $\mathrm{VO}_{2 \text { peak }}{ }^{14}$ however, there are no studies to provide evidence of the influence of resistance training with elastic tubing alone on aerobic capacity in patients with heart disease. Other studies have shown similar values when evaluating initial aerobic capacity of similar patient populations; however, in their protocols, aerobic training or combined aerobic and resistance training were performed over a prolonged period (11 months on average), before and after intervention. This indicates that aerobic capacity tends to increase significantly with long-term training. ${ }^{28}$

Analysis of quality of life in our patients did not show significant change in any of the eight SF-36 dimensions. However, it should be noted that, at the start of training, the patients already had a good perception of quality of life (i.e., values above 50 points) in seven of the eight dimensions. The exception was the physical limitation domain, which scored below 50 points on average at baseline and improved after the intervention, although the difference was not significant. The quality of life dimension is more related to functional capacity than muscle strength, as observed in a study by Nogueira et al., which showed a direct correlation between functional capacity and quality of life. ${ }^{29}$

The intervention proposed herein - resistance training with the use of elastic tubing - facilitates adaptation of the neuromuscular system to changes in intensity throughout the range of motion of the exercise, since the elastic tube generates resistance not only in the plane of movement, thus favoring constant modulation of the muscle strength used over the range of motion, but also promoting joint safety as compared to conventional exercise. This characteristic was shown to be beneficial and effective as an alternative to a conventional resistance training program within the context of a CRP. ${ }^{30}$

Limitations of this study include the small sample size and the use of a subjective variable (Borg rating of perceived exertion) as the determinant of training progression. We suggest that future studies consider other measures, such as the 1RM. In addition, future studies to measure the force exerted by elastic tubes of different diameters, which could be readily accomplished using Hooke's law, may also contribute to the literature.

\section{Conclusions}

Short-term resistance training with elastic tubing was able to promote improvement in peripheral muscle strength and functional capacity in patients with heart disease. However, no changes were observed in maximal aerobic capacity or quality of life.

\section{Potential Conflict of Interest}

No potential conflict of interest relevant to this article was reported.

\section{Sources of Funding}

This study was partially funded by CNPq.

\section{Study Association}

This study is not associated with any thesis or dissertation work.

\section{Ethics Approval and Consent to Participate}

This study was approved by the Ethics Committee of the Universidade do Oeste Paulista under the protocol number 44443915.5.0000.5515. All the procedures in 
this study were in accordance with the 1975 Helsinki Declaration, updated in 2013. Informed consent was obtained from all participants included in the study.

\section{Author Contributions}

Conception and design of the reseach: JPLN Silva. Writing of the manuscript: JPLN Silva. Acquisition of data: TIS Ferreira, GC Cvalleri, BP Galindo, NT Silva, BSA Silva. Writing of the manuscript: MMA Cruz, MR

\section{References}

1. World Health Organization. (WHO) World Stroke Organization. Global atlas on cardiovascular disease prevention and control: policies, strategies, and interventions. Geneva; 2011.

2. Brasil.Ministério da Saúde. Secretaria de Vigilância à Saúde. Plano de ações estratégicas para o enfrentamento das doenças crônicas não transmissíveis no Brasil, 2011-2022. Rio de Janeiro, DF: OMS; 2011.

3. Ribeiro AG, Cotta RMM, Ribeiro SMR. The promotion of health and integrated preventionof risk factors for cardiovascular diseases. Ciênc. Saúde Coletiva. 2012;17(1):7-17

4. Turban C, Culas C, Deley G. Effects of a short-term resistance program using elastic bands or weight machines in cardiac rehabilitation. Sci Sports. 2014;29(3):143-9.

5. Adams J, Cline M, Reed M, Masters A, Ehlke K, Hartman J. Importance of resistance training for patients after a cardiac event. Proc (Bayl Univ Med Cent). 2006;19(3):246-8

6. Muela HCS, Bassan R, Serra SM. Evaluation of the functional benefits of a cardiac rehabilitation program. Rev Bras Cardiol. 2011;24(4):241-50.

7. Guimarães FAB, Gardenghi G, Silva FMF. Reabilitação cardíaca, tratamento e prevenção: revisão bibliográfica. Rev Mov. 2015;8(1):50-9.

8. Smith SC Jr, Benjamin EJ, Bonow RO, Braun LT, Creager MA, Franklin $\mathrm{BA}$, et al. AHA/ACCF secondary prevention and risk reduction therapy for patients with coronary and other atherosclerotic vascular disease: 2011 update: a guideline from the American Heart Association and American College of Cardiology Foundation. Circulation. 2015;131(15):e408

9. Hillis LD, Smith PK, Anderson JL, Bittl JA, Bridges CR, Byrne JG, et al. 2011 ACCF/AHA Guideline for Coronary Artery Bypass Graft Surgery: executive summary: a report of the American College of Cardiology Foundation/American Heart Association Task Force on Practice Guidelines. Circulation. 2011;124(23):2610-42.

10. Melchiorri G, Rainoldi A. Muscle fatigue induced by two different resistances: Elastic tubing versus weight machines. J Electromyogr Kinesiol. 2011;21(6):954-9.

11. Thomas M, Müller T, Busse MW. Quantification of tension in Thera-Band and Cando tubing at different strains and starting lengths. J Sports Med Phys Fitness. 2005;45(2):188-98.

12. Gonçalves ACCR, Pastre CM, Camargo Filho JCS, Vanderlei LCM. Resistance exercise in heart disease: systematic review. Fisioter Mov. 2012;25(1):195-205

13. Ricci-Vitor AL, Vanderlei LCM, Pastre CM, Ramos D, Ramos EMC Ferreira Filho C, et al. Elastic tubing resistance training and autonomic modulation in subjects with chronic obstructive pulmonary disease. BioMed Res Int. 2018;2018:9573630

14. Ramos EM, Toledo-Arruda AC, Fosco LC, Bonfim R, Bertolini GN, Guarnier FA, et al. The effects of elastic tubing-based resistance training compared with conventional resistance training in patients with moderate chronic obstructive pulmonary disease: a randomized clinical trial. Clin Rehabil. 2014;28(11):1096-106.
Leite. Analysis and interpretation of the data: APCF Freire, EMC Ramos. Critical revision of the manuscript for intelectual content: LCM Vanderlei, FL Pacagnelli.

\section{Acknowledgements}

We would like to thank the National Council for Scientific and Technological Development (CNPq) process: 800098/2014-5 for the award of an undergraduate research scholarship (PIBIC).

15. Brooks D, Solway S, Gibbons WJ. ATS statement on six-minute walk test Am J Respir Crit Care Med. 2003;167(9):1287.

16. Meneghelo RS, Araújo CGS, Stein R, Mastrocolla LE, Albuquerque PF, Serra SM. III Diretrizes da Sociedade Brasileira de Cardiologia sobre teste ergométrico. Arq Bras Cardiol. 2010;95(5):1-26.

17. Kendrick KR, Baxi SC, Smith RM. Usefulness of the modified 0-10 Bor scale in assessing the degree of dyspnea in patients with COPD and asthma. J Emerg Nurs. 2000;26(3):216-22.

18. Kuipers H, Verstappen FTJ, Keizer HA, Guerten P, Van Kranenburg G. Variability of anaerobic performance in the laboratory and its physiologic correlates. Int J Sports Med. 1985;6(4):197-201.

19. Ciconelli RM, Ferraz MB, Santos W, Meinão I, Quaresma MR. Tradução para a língua portuguesa e validação do questionário genérico de avaliação de qualidade de vida SF-36 (Brasil SF-36). Rev Bras Reumatol. 1999;39(3):143-50.

20. Tavares A, Brandão AA, Sanjuliani AF, Nogueira AR, Machado CA, Polide-Figueiredo, et al., Sociedade Brasileira de Cardiologia. VI Diretrizes Brasileiras de Hipertensão Arterial. Arq Bras Cardiol. 2010;95(1 supl 1):1-51.

21. Karvonen M, Kentala E, Mustata O. The effects of training on heart rate Ann Med Exp Biol Fenn. 1957;35(3):307-15.

22. Fardy PS, Franklin BA, Porcari JP, Verrill DE. Técnicas de treinamento em reabilitação cardíaca. São Paulo: Manole; 2001. 149p.

23. Borg GAV. Psychophysical bases of perceived exertion. Med Sci Sport Exerc. 1982;14(5):377-81

24. Granacher U, Lesinski M, Büsch D, Muehlbauer T, Prieske O, Puta C, et al. Effects of resistance training in youth athletes on muscular fitness and athletic performance: a conceptual model for long-term athlete development. Front Physiol. 20169 May;7:164.

25. Persch LN, Ugrinowitsch C, Pereira G, Rodacki AL. Strength training improves fall-related gait kinematics in the elderly: a randomized controlled trial. Clin Biomech. 2009;24(10):819-25.

26. American College of Sports Medicine. American College of Sports Medicine position stand. Progression models in resistance training for healthy adults. Med Sci Sports Exerc. 2009;41(3):687-708

27. Simões RP, Mendes RG, Castello V, Machado HG, Almeida LB, Baldissera $\mathrm{V}$, et al. Heart-rate variability and blood-lactate threshold interaction during progressive resistance exercise in healthy older men. J Strengh Cond Res. 2010;24(5):1313-20.

28. Marzolini S, Mertens DJ, Oh PI, Plyley MJ. Self reported compliance to home-based resistance training in cardiac patients. Eur J Cardiovasc Prev Rehabil. 2010;17(1):35-41.

29. Nogueira IDB, Servantes DM, Nogueira PAMS, Pelcerman A, Salvetti $\mathrm{XM}$, Salles F, et al. Correlation between quality of life and functional capacity in heart failure. Arq Bras Cardiol. 2010;95(2):238-43.

30. Guzun R, Aguilaniu B, Wuyam B, Mezin P, Koechlin-Ramonatxo C, Auffray C, et al. Effects of training at mild exercise intensities on quadriceps muscle energy metabolism in patients with chronic obstructive pulmonary disease. Acta Physiol. 2012;205(2):236-46. 